\title{
T-helper cell type-1 transcription factor T-bet is upregulated in pulmonary sarcoidosis
}

\author{
E. Kriegova*, R. Fillerova*, T. Tomankova*, B. Hutyrova\#, F. Mrazek*, T. Tichy \\ V. Kolek ${ }^{\#}$, R.M. du Bois ${ }^{+}$and M. Petrek*
}

ABSTRACT: Upregulation of genes for interferon (IFN)- $\gamma$ and CXC chemokine receptor (CXCR)3 expression, two crucial molecules in sarcoid inflammation and granuloma formation, is directly controlled by the T-helper (Th) 1 transcription factor T-bet (T-box, expressed in T-cells). However, there is no information on T-bet expression in sarcoidosis or its relationship with "sarcoidosisassociated" genes.

Therefore, we investigated expression of T-bet mRNA and, in parallel, a spectrum of genes known to be involved in sarcoidosis pathogenesis. Transcripts were determined in bronchoalveolar lavage (BAL) cells from 62 sarcoidosis patients and 25 controls by quantitative RT-PCR; T-bet protein was localised by immunohistochemistry.

Patient's BAL cells expressed higher mRNA T-bet levels than those of controls (mean \pm SD fold change $3.64 \pm 1.72 ; p=0.00006)$. T-bet $m R N A$ expression did not vary between clinical phenotypes as assessed by chest radiography stage, presence/absence of Löfgren's syndrome, extrapulmonary/pulmonary involvement or progressing/remitting disease $(p>0.05)$. T-bet mRNA expression correlated with expression of IFN- $\gamma, \mathrm{CC}$ chemokine ligand 5, CXC chemokine ligand (CXC)10, interleukin (IL)-2 receptor/IL-15 receptor $\beta$, CXCR3 and CXCR6 $(p<0.01)$. T-bet protein was localised to alveolar macrophages and lymphocytes, tissue multinucleated giant cells, macrophages and lymphocytes.

In pulmonary sarcoidosis, T-bet upregulation is associated with changes in expression of IFN- $\gamma$, CXCR3 and chemokines/receptors involved in the pathogenesis of sarcoidosis, which suggests a role for T-bet in this Th1 disease, including modulation of some sarcoidosis-associated genes.

KEYWORDS: CC chemokine ligand 5, chemokines, CXC chemokine receptor-3, CXC chemokine receptor-6, interferon- $\gamma$, lung inflammation

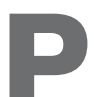

ulmonary sarcoidosis is an inflammatory disorder characterised by the accumulation of activated lymphocytes and macrophages in the lung, resulting in the formation of granulomas [1, 2]. A key role in granuloma formation is played by interferon (IFN) $-\gamma$ [3] and Thelper (Th) type-1 cells accumulating in the sarcoid lungs, which express chemokine receptor CXC chemokine receptor (CXCR)3 [4]. There is evidence that both CXCR3 and IFN- $\gamma$ are under the direct control of a Th1 transcription factor, T-bet (T-box, expressed in T-cells) [5-7]. Moreover, this key Th1 transcription factor, T-bet, directs Th1 lineage commitment [8]. Although sarcoidosis is a predominantly Th1 immune disease $[9,10]$, and CXCR3 and IFN- $\gamma$ are implicated in its pathogenesis, there is currently no information about the involvement of the Th1 transcription factor T-bet in this disorder.

In addition to T-bet controlling CXCR3 and IFN- $\gamma$ expression, it has also been involved in the regulation of numerous immune system genes. In contrast to the direct requirement of T-bet for CXCR3 and IFN- $\gamma$ expression irrespective of cell type, regulation of other genes may be dependent on the cell type and the extent of their regulation may vary [5]. For example, T-bet induces modest activation of interleukin (IL)-2 receptor (2R) $\beta$ (CD122) and CC chemokine ligand (CCL)3 depending on the cell type [5]. The relationship between T-bet and the expression of other chemokines/receptors in different cell types/diseases has also been reported, such as CCL5 in peripheral blood from asthmatics [11], CCR5 and CXCR4 in endometrial T-cells [12], and CCL5 and CXC chemokine ligand (CXCL) 9 in experimental crescentic glomerulonephritis [13]. However, knowledge on T-bet targets, namely gene promoters under its functional regulation and relevant regulatory mechanisms, is far from complete. To address the role for T-bet as a plausible regulator of immune mediators in sarcoidosis, we studied its
AFFILIATIONS

*Laboratory of Immunogenomics and Immunoproteomics,

Depts of ${ }^{\#}$ Respiratory Medicine, and "Clinical and Molecular Pathology, Faculty of Medicine and Dentistry,

Palacky University, Olomouc, Czech Republic.

${ }^{+}$National Jewish Health, Denver, CO, USA.

CORRESPONDENCE

M. Petrek

Laboratory of Immunogenomics and Proteomics

Palacky University

I.P. Pavlova 6

CZ-77520

Olomouc

Czech Republic

E-mail: petrekm@fnol.cz

Received:

June 102010

Accepted after revision:

March 282011

First published online:

May 032011

European Respiratory Journal

Print ISSN 0903-1936

Online ISSN 1399-3003 
TABLE 1 Clinical and laboratory data of investigated sarcoidosis patients

\begin{tabular}{|c|c|c|c|c|c|}
\hline Characteristics & Sarcoidosis & CXR stage I & CXR stage II & CXR stage III & CXR stage IV \\
\hline Subjects & 62 & 17 & 33 & 8 & 4 \\
\hline Males/females & $26 / 36$ & $9 / 8$ & $14 / 19$ & $3 / 5$ & $0 / 4$ \\
\hline Smoking yes/no/ex & $6 / 43 / 13$ & $3 / 11 / 3$ & $3 / 21 / 9$ & $0 / 7 / 1$ & $0 / 4 / 0$ \\
\hline CXR stages $\mathrm{I} / \mathrm{II} / \mathrm{III} / \mathrm{IV}$ & $17 / 33 / 8 / 4$ & 17 & 33 & 8 & 4 \\
\hline Macrophages \% & $74.5 \pm 13.0(40.0-97.1)$ & $75.1 \pm 12.4(49.4-91.1)$ & $75.2 \pm 12.6(40.0-97.1)$ & $73.3 \pm 17.6(44.4-92.1)$ & $68.9 \pm 13.4(56.0-82.7)$ \\
\hline Lymphocytes \% & $22.8 \pm 12.3(2.0-54.0)$ & $24.0 \pm 12.1(8.0-49.0)$ & $21.7 \pm 11.7(2.0-47.0)$ & $24.6 \pm 17.9(7.6-54.0)$ & $22.7 \pm 5.7(16.7-28.0)$ \\
\hline Neutrophils \% & $2.0 \pm 3.6(0.0-18.0)$ & $0.6 \pm 0.7(0.0-2.3)$ & $2.1 \pm 3.7(0.0-18.0)$ & $2.0 \pm 3.4(0.0-10.0)$ & $7.1 \pm 6.4(0.6-14.0)$ \\
\hline Eosinophils \% & $0.7 \pm 2.3(0.0-17.0)$ & $0.3 \pm 0.4(0.0-1.3)$ & $1.0 \pm 3.0(0.0-17.0)$ & $0.2 \pm 0.4(0.0-1.0)$ & $1.8 \pm 2.8(0.0-5.0)$ \\
\hline $\mathrm{CD} 3+\%$ & $86.1 \pm 11.8(36.0-98.0)$ & $91.1 \pm 4.9^{\#}(79.0-97.0)$ & $85.1 \pm 11.4(51.0-98.0)$ & $87.4 \pm 9.7(67.0-96.0)$ & $72.5 \pm 25.0(36.0-92.0)$ \\
\hline $\mathrm{CD} 4+\%$ & $67.8 \pm 17.9(23.0-92.0)$ & $78.7 \pm 9.4^{\#}(54.0-92.0)$ & $64.7 \pm 18.5(23.0-87.0)$ & $66.6 \pm 19.6(33.0-90.0)$ & $52.5 \pm 19.7(30.0-74.0)$ \\
\hline
\end{tabular}

Data are presented as $n$ or mean \pm SD (range). CXR: chest radiograph; LS: Löfgren's syndrome; BAL: bronchoalveolar lavage. ${ }^{\#}:$ T-cell population data were not available for one subject.

relationship with cytokines and their receptors that have been previously implicated in the pathogenesis of sarcoidosis. Of these "sarcoidosis-associated" genes we mainly focused on chemokines directing the trafficking of activated T-cells into sarcoid lungs, such as CCL2, CCL5, CXCL9, CXCL10 and CXCL11 [14-19], and chemokine receptors, CC chemokine ligand (CCR)5 [10] and CXCR6 [4] preferentially expressed on activated lymphocytes.

First, we determined the expression of T-bet in bronchoalveolar lavage (BAL) cells obtained from 62 sarcoidosis patients and 25 control subjects and searched for differences in its expression patterns in patient subgroups with distinct disease phenotypes. Secondly, we aimed to address the question as to whether there is evidence supporting the theory of T-bet modulation of CXCR3 and IFN- $\gamma$ gene expression in sarcoid lungs by performing correlation analyses of their mRNA expression profiles in BAL cells. Finally, we investigated the relationship of $\mathrm{T}$-bet with a range of sarcoidosis-associated genes, namely: 1) ligands of CXCR3 receptor (CXCL9, CXCL10 and CXCL11) [14-17]; 2) chemokines CCL2 and CCL5 [18, 19]; 3) chemokine receptors CCR2, CCR5 and CXCR6 [4, 10, 20, 21]; and 4) $\beta$-chain subunit of IL-2R and IL-15R receptors [10, 22]. Herein, we report upregulation of T-bet in sarcoidosis and its relationship with some sarcoid-associated molecules. Some of our data have been presented previously [23].

\section{MATERIALS AND METHODS \\ Subjects}

BAL was performed according to a standard protocol [24] in 62 patients with pulmonary sarcoidosis (chest radiograph (CXR) stage I $n=17$; stage II $n=33$; stage III $n=8$; stage IV $n=4$ ) and 25 control subjects of Czech origin. The diagnosis of sarcoidosis was determined in compliance with international criteria [25]. Because radiographic staging provides limited information on prognosis and no information on disease activity and in order to exclude the influence of smoking on the gene expression, we further subdivided 56 nonsmoking patients according to the duration of symptoms as assessed by: follow-up at 2 yrs (remission $n=23$, progression persistence $n=26$; in seven patients, data after 2 yrs were not available); presence/absence of Löfgren's syndrome (LS; $n=8 / 48$ ); and BAL cellular profile (patients with $>11 \%$ BAL lymphocytes $n=45$; patients with $\leqslant 11 \%$ BAL lymphocytes $n=11$; patients with $\geqslant 85 \%$ BAL macrophages $n=11$; patients with $<85 \%$ BAL macrophages $\mathrm{n}=45)$. The reference values for BAL cell counts $(\geqslant 85 \%$ macrophages, $\leqslant 11 \%$ lymphocytes, $\leqslant 2.5 \%$ neutrophils and $\leqslant 1 \%$ eosinophils) were based on our own laboratory values and correspond to ZiEgEnHAGEN et al. [26]. Further subgroups were formed according to organ involvement (pulmonary involvement only $n=37$; extrapulmonary involvement $\mathrm{n}=19$ ). For clinical and laboratory characteristics of the investigated patients, see table 1 . The control group consisted of 25 subjects (17 nonsmokers (nine males and eight females) and eight smokers (seven males and one female); mean \pm SD age $44.7 \pm 16.2$ yrs) undergoing BAL as part of clinical investigation for psychogenic cough, cough associated with gastro-oesophageal reflux disease or lung hypertension. In this group, three patients with suspected pulmonary malignancy were included; lavage was performed in an unaffected lobe. All had normal BAL fluid cytology, immunology, microbiology and CD4+/CD8+ ratio. No patient received corticosteroid treatment before BAL.

All patients gave their informed consent for the use of BAL, which was obtained primarily for diagnostic evaluation, for the purpose of this study. The local ethical committee of Palacky University and Faculty Hospital Olomouc (Olomouc, Czech Republic) approved the study.

\section{BAL sample processing and RNA isolation}

BAL cells were separated from the fluid by centrifugation as described previously [21]. Total RNA from unseparated BAL cells was isolated with the mirVana miRNA kit (Ambion, Austin, TX, USA). RNA quality and quantity was measured 
using the 2100 Bioanalyzer with RNA 6000 Nano assays (Agilent Technologies, Palo Alto, CA, USA). Reverse transcription was performed with Reverse-i $\mathrm{T}^{\mathrm{TM}}$ RTase Blend using anchored deoxythymidine primers (ABgene, Epsom, UK) according to the manufacturer's instructions.

\section{Measurement of mRNA expression profiles by quantitative RT-PCR}

The primer sequences, probes and amplicon sizes for investigated genes are listed in table 2. PCR reactions were performed on RotorGene3000 system (Corbett Research, Sydney, Australia) as described previously [27]. The relative expression was calculated by a second-derivative method (RotorGene Software 6.1.81; Corbett Research). cDNA from human universal reference RNA (Stratagene, La Jolla, CA, USA) was used as a calibrator and $P S M B 2$ as a reference gene [27]. Changes in expression levels are presented as a mean $\pm \mathrm{SD}$ fold change of relative expression compared to control subjects (normalised to 1.0) [28, 29].

\section{Determination of cell-associated T-bet protein by immunohistochemistry}

The cryostat slices of paraffin-embedded lung tissues obtained by transbronchial biopsy $(n=5)$, video-assisted thoracoscopic surgery $(n=3)$ and lymph node sample $(n=1)$ were processed by immunohistochemistry. Briefly, after microwave antigen retrieval in a citrate buffer ( $\mathrm{pH}$ 6.0), the slides were incubated with anti-human T-bet monoclonal antibody (clone 525803, dilution 1:50; R\&D Systems Inc., Minneapolis, MN, USA) and visualised using a standard indirect avidin-biotin horseradish peroxidase method (EnVision+ System-HRP; Dako, Glostrup, Denmark). Sections were counterstained with haematoxylin. Tbet staining was compared with that of mouse immunoglobulin (Ig)G isotype control antibody (clone DAK-GO1, dilution 1:50; Dako) for all cases studied to confirm staining specificity. The type of lymphocytes expressing T-bet was identified by subpopulation-specific antibodies (CD3, clone F7238; CD 20, clone L26; dilution 1:50; Dako).

\section{Determination of cell-associated T-bet protein by immunocytochemistry}

T-bet protein was detected on the acetone-chloroform fixed cytocentrifuge preparations of BAL cells using the streptavidin-biotin/horseradish peroxidase method using an anti-T-bet antibody (clone 525803, dilution 1:50; R\&D Systems Inc.) and a negative control mouse IgG1 (clone DAK-GO1, dilution 1:50; Dako) as described previously [16].

TABLE 2 Description of investigated genes, used primers, locked nucleic acid (LNA) probes and amplicon sizes in RT-PCR

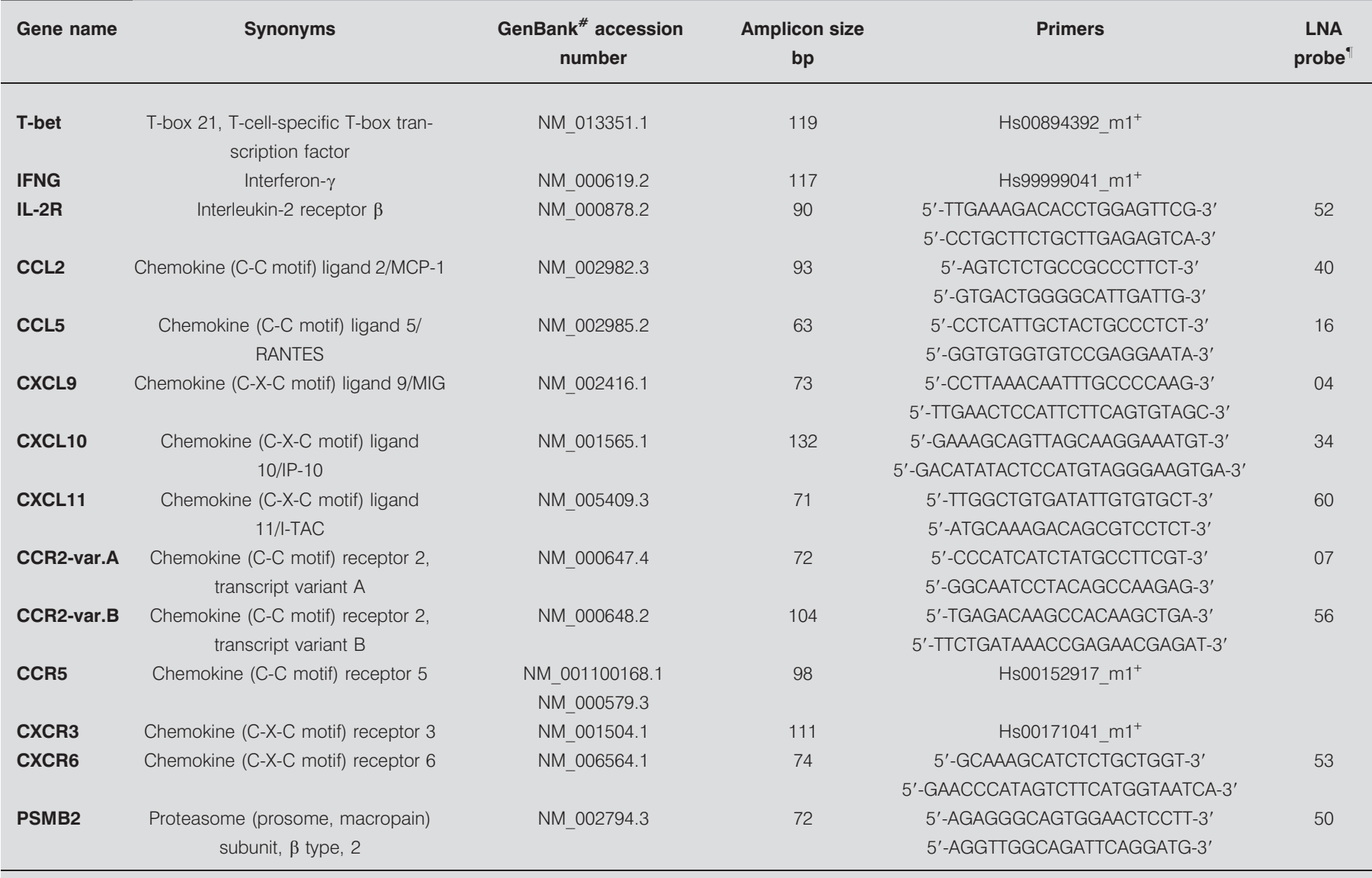

RANTES: regulated on activation, normal T-cell expressed and secreted. ${ }^{*}$ : gene sequences available online at www.ncbi.nIm.nih.gov; ": numbers of LNA probes according to the commercially available library (www.universalprobelibrary.com), primers were designed using ProbeFinder assay design tool (Roche Applied Science, Indianapolis, IN, USA); +: primers/probe sets (Assays-on-Demand) were from Life Technologies Corporation (Carlsbad, CA, USA). 


\section{Statistical analysis}

Comparisons of mRNA expression among two groups were performed using the nonparametric Mann-Whitney U-test and for three or more groups by the Kruskal-Wallis test. Spearman's rank correlation was used to assess the relationships between T-bet and chemokine/receptor mRNA expressions, cellular profile of BAL fluid and the clinical course of sarcoidosis. All calculations were performed by software SPSS 16.0 (SPSS Inc., Chicago, IL, USA). A p-value $<0.05$ was considered as significant.

\section{RESULTS}

mRNA expression profiles of T-bet and selected mediators as a whole and in specific sarcoid clinical phenotypes

In order to investigate the mRNA expression levels of $\mathrm{T}$ bet, IFN- $\gamma$, IL-2R/IL-15R $\beta$, CCL2, CCL5, CXCL9, CXCL10, CXCL11, CCR2, CCR5, CXCR3 and CXCR6 in sarcoidosis, the relative mRNA expression levels in BAL cells were determined in control subjects, sarcoidosis patients and patient subgroups. All RNA samples from patients and controls were of good quality (relative integrity number $>5$ ).

mRNA expression profiles of transcription factor T-bet as a whole and sarcoid clinical phenotypes

The relative expression level of T-bet was higher in sarcoidosis patients than in control subjects (mean \pm SD fold change $3.64 \pm 1.72 ; p=0.00006$ ) (fig. 1a). When we compared T-bet expression in smoking and nonsmoking (ex-smokers and nonsmokers) patients and controls, we observed trend to lower T-bet expression in smoking controls $(p=0.06)$ and smoking patients $(\mathrm{p}=0.12)$ than in corresponding nonsmoking controls and patients, respectively (fig. 1b). Therefore, to exclude the influence of smoking, we only compared nonsmoking patients subdivided by the sarcoid clinical phenotypes.

When the expression level of T-bet was compared within specific CXR stages (I, II, III and IV), T-bet expression did not vary between stages: $4.00 \pm 1.87,3.00 \pm 1.40,2.22 \pm 1.04$ and $5.68 \pm 2.65$, respectively ( $\mathrm{p}>0.05$ ) (fig. $1 \mathrm{c})$. When T-bet expression levels were compared in subgroups according to the presence/absence of LS (phenotype characterised by an acute onset, fever, erythema nodosum, arthralgias and bilateral hilar lymphadenopathy), no difference was observed between LS $(2.68 \pm 1.22)$ and non-LS patients $(3.44 \pm 1.57 ; \mathrm{p}>0.05)$ (fig. $1 \mathrm{~d})$. Similarly, when patients were subdivided according to the involved organs, the difference between T-bet expression in patients with only lung involvement $(3.86 \pm 1.76)$ and in those who also had extrapulmonary involvement $(2.30 \pm 1.05)$ was not significant $(\mathrm{p}>0.05)$ (fig. 1e). Also, in patients subgrouped according to disease outcome after 2-yr follow-up, we did not observe any difference in the number of T-bet transcripts (disease remission $2.71 \pm 1.23$, progressive/persistent sarcoidosis 3.24 $\pm 1.47 ; \mathrm{p}>0.05$ ) (fig. 1f).

Further patient subgroups were formed according to the cellular profile of the BAL. In subgroups based on BAL macrophage counts (cut-off $85 \%$ macrophages), patients with BAL macrophages within the normal range expressed lower levels of T-bet $(1.73 \pm 0.79)$ than patients with $<85 \%$ BAL macrophages $(3.72 \pm 1.69, \mathrm{p}=0.05)$ (fig. $1 \mathrm{~g})$. Also, in subgroups based on BAL lymphocyte counts (cut-off $11 \%$ lymphocytes), Tbet expression in patients with high BAL lymphocytes tended to exhibit higher T-bet expression $(3.66 \pm 1.66)$ than those with lower BAL lymphocytes $(1.98 \pm 0.90, \mathrm{p}=0.15)$ (fig. $1 \mathrm{~h})$.

\section{Correlation of T-bet mRNA expression with gene expression of selected sarcoidosis-relevant genes}

Because T-bet was identified as a potent modulator of the mRNA expression of numerous genes, we investigated its possible relationship with the mRNA expression of IFN- $\gamma$, IL-2R/IL15R $\beta$, CCL2, CCL5, CXCL9, CXCL10, CXCL11, CCR2, CCR5, CXCR3 and CXCR6 in BAL cells from sarcoidosis patients.

Expression of T-bet mRNA correlated with elevated expression of IFN- $\gamma \quad(p<0.001)$, CCL5 $(p<0.001)$, CXCL9 $(p=$ 0.033), CXCL10 ( $p=0.006)$, IL-2R/IL-15R $\beta(p<0.001), C X C R 3$ $(\mathrm{p}<0.001)$ and CXCR6 $(\mathrm{p}=0.001)$ (table 3$)$. Although we also observed a positive correlation with CCR2-variant $B(p=0.003)$, this transcript variant is not upregulated in sarcoidosis when compared with controls. The expression of CCR2-variant A, which was found to be upregulated in our sarcoid patients, did not correlate with T-bet $(p>0.05)$. Similarly, our correlation analysis showed that expression of CCL2, CXCL11 and CCR5 $(\mathrm{p}>0.05)$ were not associated with T-bet. Spearman's coefficients for all studied genes are listed in table 3.

Expression of mRNA for genes (expressed as a fold change) found to be associated with T-bet in patient subgroups presenting with/without LS are shown in figure 2a. Out of the genes correlated with T-bet, mRNA expression of CCL5 $(p=0.033)$, CXCL9 $(p=0.041)$ and IL2-R/IL-15R $\beta(p=0.042)$ was higher in our patients presenting without LS in comparison to those with LS. Expression of chemokine CXCL10 tended to be higher in patients without LS $(p=0.08)$; for IFN- $\gamma(p=0.25)$ and the receptors CXCR3 $(p=0.12)$ and CXCR6 $(p=0.22)$, the difference between both groups did not reach significance.

Based on the significant differences in mRNA expression profile of T-bet in patient subgroups according to BAL macrophages (cut-off 85\%), we also compared mRNA expression of chemokines CCL5 and CXCL10, produced mainly by macrophages, in these subgroups. A higher number of CCL5 transcripts was detected in patient subgroups with low BAL macrophages in comparison to patients with high BAL macrophages $(p=0.003)$; the difference between the number of CXCL10 transcripts between both subgroups did not reach significance $(p=0.17)$ (fig. $2 b)$.

\section{Correlation of T-bet $m R N A$ expression with BAL cellular profile}

In order to find out whether T-bet mRNA expression correlates with the BAL cellular profile, we performed correlation analysis. T-bet mRNA expression correlated with relative (Spearman's rho $\left.\left(\mathrm{r}_{\mathrm{s}}\right)=0.427, \mathrm{p}<0.001\right)$, but not with absolute $\left(r_{s}=0.008, p>0.05\right)$, BAL lymphocyte count. Moreover, T-bet negatively correlated with relative $\left(r_{s}=-0.381, p<0.002\right)$, and tended to correlate with absolute $\left(r_{s}=-0.236, p=0.07\right)$, BAL macrophages. No association was observed with BAL neutrophils or eosinophils $(\mathrm{p}>0.05)$.

\section{Determination of cell-associated T-bet protein}

To identify the cellular source of T-bet, cytocentrifuge preparations of BAL cells, lung and lymph node biopsies from patients with sarcoidosis were immunostained for T-bet 

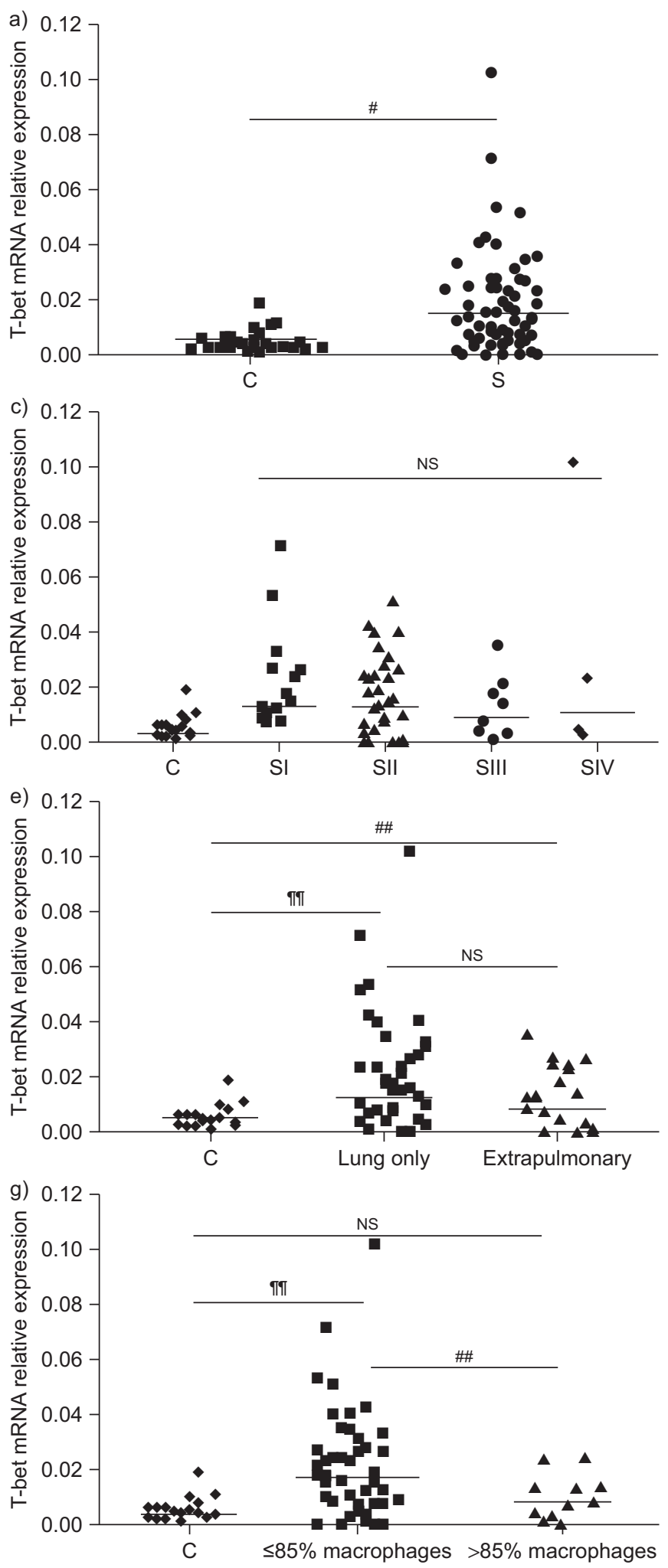
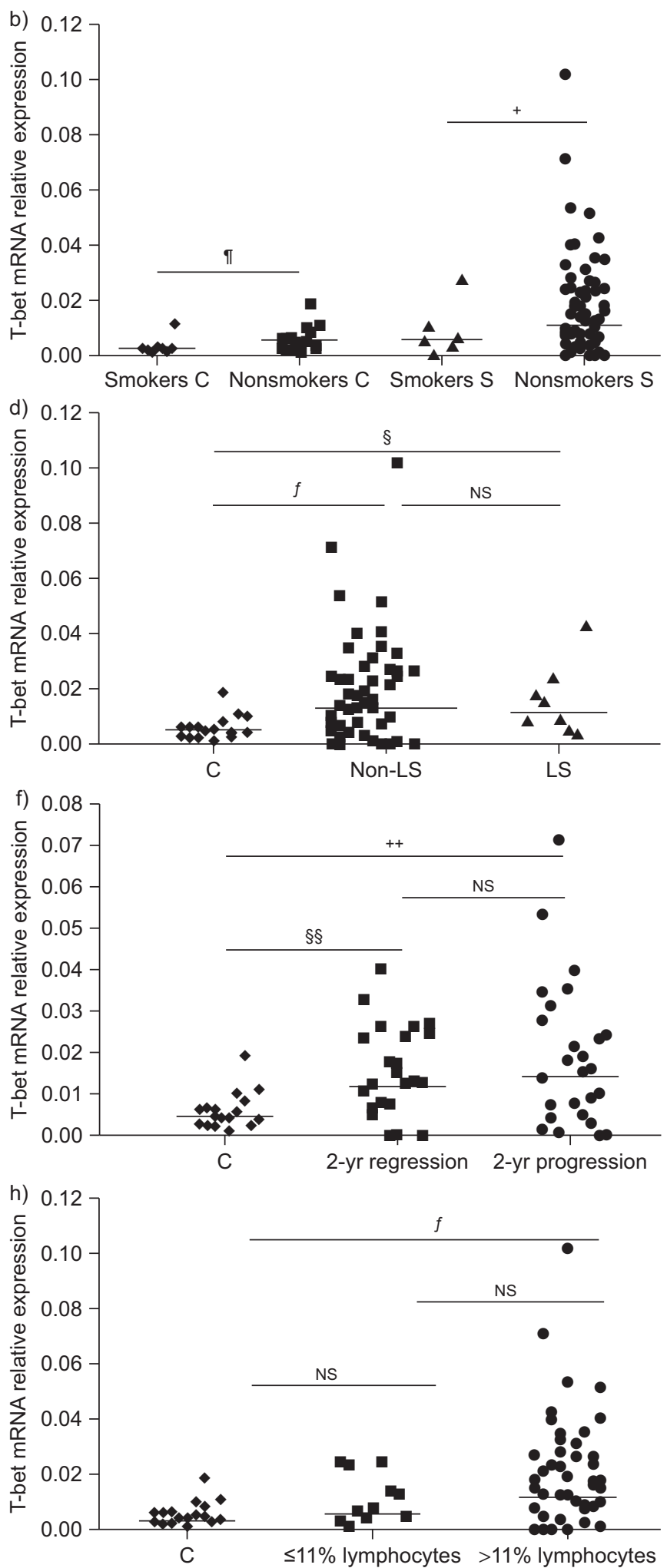

FIGURE 1. Distribution of relative T-bet (T-box, expressed in T-cells) mRNA expressions (ratio target gene/reference PSMB2 gene) in unseparated bronchoalveolar lavage (BAL) cells in a) 62 sarcoidosis patients (S) and 25 control subjects (C) and in b) subgroups based on smoking status. Further subanalysis was performed in nonsmoking sarcoidosis patients divided according to: c) chest radiograph stages (I, II, III or IV); d) the presence or absence of Löfgren's syndrome (LS); e) involved organs (pulmonary versus extrapulmonary); f) disease outcome after 2 yrs (regression versus progression); g) BAL macrophage counts (cut-off 85\%); and h) BAL lymphocyte counts (cut-off 11\%). Group medians are indicated by horizontal bars. For more details on quantitative RT-PCR conditions and calculation of relative expression see Materials and

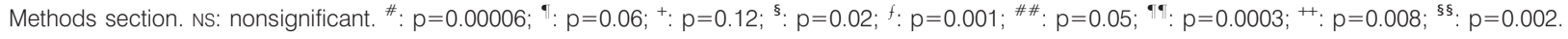




\begin{tabular}{|c|c|c|c|c|}
\hline TABLE 3 & $\begin{array}{l}\text { nalysis of T-bet (T-box, expresse } \\
\text { enes }\end{array}$ & in T-cell) expression & express & sarcoidosis- \\
\hline \multirow[t]{2}{*}{ Gene } & \multirow[t]{2}{*}{ Sarcoidosis versus controls $p$-value } & \multicolumn{2}{|c|}{ Correlation with T-bet expression } & \multirow[t]{2}{*}{ [Ref. $]^{\#}$} \\
\hline & & Spearman's coefficient & p-value & \\
\hline IFNG & $0.00002^{* *}$ & 0.660 & $<0.001^{\star *}$ & {$[3,30,31]$} \\
\hline CCL5 & $0.0002^{\star *}$ & 0.627 & $<0.001^{* *}$ & {$[18,19]$} \\
\hline CXCL9 & $0.0007^{* *}$ & 0.278 & $0.033^{*}$ & {$[14,15]$} \\
\hline CXCL10 & $0.0006^{\star *}$ & 0.353 & $0.006^{* *}$ & {$[14,16,17]$} \\
\hline CXCL11 & $0.013^{*}$ & 0.259 & 0.052 & {$[14,15]$} \\
\hline CCR2-variant A & $0.003^{* *}$ & 0.232 & 0.116 & {$[20]$} \\
\hline
\end{tabular}

protein. On lung biopsies, T-bet was expressed by macrophages, multinucleated giant cells, epithelioid macrophages of the granuloma and lymphocytes infiltrating the biopsy specimen of sarcoidosis patients (fig. 3a-d). Of lymphocytes, T-bet was only expressed in T-cells. In the lymph node of one sarcoidosis patient, T-bet was highly expressed in T-cells (fig. 3e and 1). In cytocentrifuge preparations of BAL cells from patients with sarcoidosis, T-bet protein was detected in alveolar macrophages and alveolar lymphocytes (fig. 3f).

\section{DISCUSSION}

Because the Th1 transcription factor T-bet has been shown to play a pivotal role in controlling Th1 responses and in the transcription of CXCR3 and IFN- $\gamma$, two crucial genes for sarcoidosis pathogenesis, we analysed T-bet mRNA and protein expression in BAL cells from sarcoidosis patients. In comparison with control subjects, T-bet mRNA expression was elevated in sarcoidosis, thus, suggesting a role for this transcription factor in the immunopathogenesis of this Th1 disease. T-bet protein expression was localised to alveolar macrophages and lymphocytes, and tissue multinucleated giant cells, epithelioid macrophages and lymphocytes. Finally, our experiments investigating possible relationships between T-bet and a range of genes involved in the pathogenesis of sarcoidosis showed that T-bet is associated with the increased mRNA expression of IFN- $\gamma$, CCL5, CXCL10, CXCR3, CXCR6 and IL-2R/15Rß.

In view of the crucial role of T-bet in Th1 differentiation [8] and its requirement for CXCR3 and IFN- $\gamma$ upregulation [5-7], we hypothesised that T-bet is involved in the pathogenesis of the Th1-polarised inflammation in sarcoidosis. In line with this hypothesis, our data showed that T-bet mRNA expression is elevated in sarcoidosis patients when compared to control subjects. Increased levels of T-bet have also been reported in other Th1 diseases, such as Crohn's disease [32] and coeliac disease $[33,34]$, where T-bet expression paralleled the disease
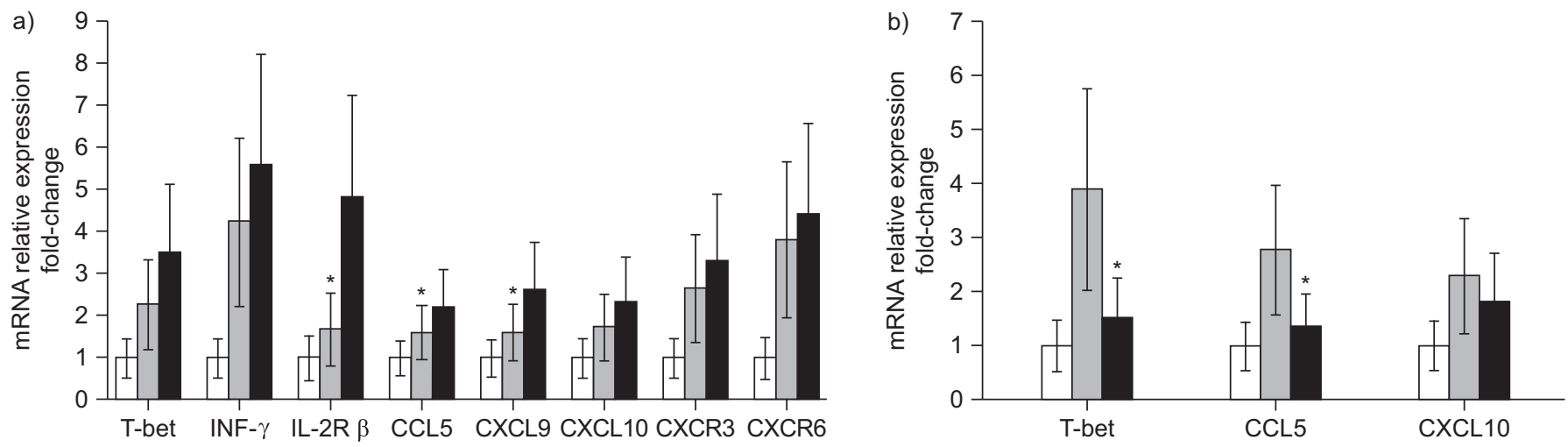

FIGURE 2. Comparison between the relative mRNA expression of genes interferon (IFN)- $\gamma$, interleukin-2 receptor (IL-2R) $\beta, C C L 5, C X C L 9, C X C L 10, C X C R 3$ and CXCR6 associated with T-bet (ratio target gene/reference PSMB2 gene) in unseparated bronchoalveolar (BAL) cells in a) patients presenting with ( $\square$ ) and without ( $\square$ ) Löfgren's syndrome and b) patients with $<85 \%$ BAL macrophages $(\square)$ and $\geqslant 85 \%$ BAL macrophages ( $\square$ ). Data are presented as a mean fold-change of relative expression in the patients related to the expression in control subjects set as $1.0(\square)$. Whiskers represent the SD values. *: $p<0.05$ for levels between patient subgroups. 

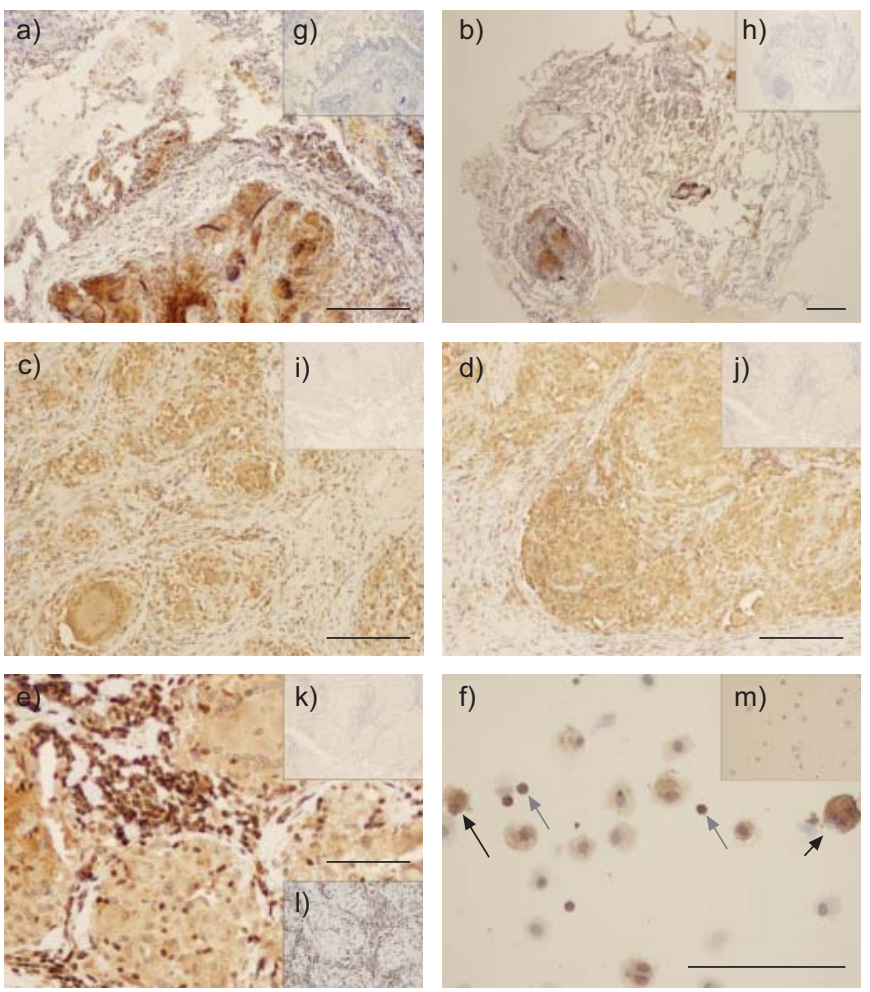

FIGURE 3. Detection of T-bet protein in lung and lymph node sections and in bronchoalveolar lavage (BAL) cells from patients with sarcoidosis. a-d) In four representative patients with sarcoidosis, T-bet protein was expressed by epithelioid macrophages of the granuloma, macrophages, multinucleated giant cells and lymphocytes in lung tissues. e) In the lymph node specimen obtained from a sarcoidosis patient, T-bet was expressed in high intensity by T-cells (insert I) (CD3 antibody staining). g-k) Serum isotype controls with haematoxylin counterstain. On BAL cytospins (representative example), positively stained cells were observed after incubation with f) anti-T-bet-antibody, but not with $m$ ) the irrelevant antibody. T-bet protein expression was localised predominantly to the cytoplasm of BAL macrophages (black arrows) and lymphocytes (grey arrows). a, e, f) Scale bars $=100 \mu \mathrm{m}$; b-d) scale bars $=200 \mu \mathrm{m}$.

course. In line with the observation by NizRI et al. [35], we observed a trend to lower expression of T-bet in smokers when compared to nonsmokers in control and patient groups. For this reason, we further subanalysed the T-bet expression only in nonsmoking subjects ( $90 \%$ of patients). In the case of sarcoidosis phenotypes assessed by CXR staging, we did not observe any difference with individual CXR stages. Furthermore, we did not detect any difference between patients presenting with/without LS, with extrapulmonary/ pulmonary involvement and progressing/remitting disease. This allowed us to speculate that T-bet might not have an influence on the clinical phenotype, but it may act as a "sarcoidosis-predisposing" molecule, thus, explaining the relatively lower incidence of sarcoidosis in smokers [36].

Based on observations in T-bet-deficient mice and transfected cell lines that T-bet is an absolute requirement for the induction of transcription of CXCR3 and IFN- $\gamma$ in Th1 cells [5-7], we proposed that T-bet may also modulate CXCR3 and IFN- $\gamma$ gene expression in sarcoidosis. The T-bet control of CXCR3 expression is of particular interest in sarcoidosis because this chemokine receptor plays a pivotal role in recruiting T-cells to the lungs with subsequent granuloma formation [4]. In this study, we observed a strong correlation between T-bet and CXCR3 and IFN- $\gamma$ expression in BAL cells obtained from sarcoid lungs. Our correlation analysis, therefore, supports the concept of T-bet modulation of CXCR3 and IFN- $\gamma$ gene expression in sarcoid lung. Further functional studies are needed to confirm the direct control of CXCR3 and IFN- $\gamma$ expression by T-bet in sarcoid lung.

Although T-bet has been involved in the regulation of numerous immune system-associated genes, knowledge about its targets, promoter motives and regulatory mechanisms is far from complete. In contrast to the direct requirement of T-bet for CXCR3 and IFN- $\gamma$ expression irrespective of cell type, regulation of other genes may be highly "context" dependent [5]. To address the role for T-bet in sarcoidosis from this aspect, we studied its relationship with selected cytokines/receptors implicated in disease pathogenesis. Of the studied chemokine receptors, we observed a strong correlation between $\mathrm{T}$-bet and CXCR6, a receptor co-expressed on CXCR3 T-cells in sarcoidosis [4]. Furthermore, a strong correlation was observed between T-bet and CCL5 and CXCL10, two chemokines that play an important role in the recruitment of T-cells to the sarcoid lungs $[4,15-17,19]$. Additionally, we observed a strong association between T-bet and IL-2R/IL-15R $\beta$ (CD122) in our sarcoidosis patients; this association has been already reported in animal cell cultures $[5,7]$. The IL-2R/IL-15R $\beta$ subunit is shared by two sarcoidosis-related receptors [37]: activation marker IL-2R and IL-15R, a receptor involved in the growth of sarcoid T-cells $[10,22]$.

We were also interested to investigate which cell type(s) express T-bet. Because the chemokines/receptors, expression of which has been shown to be modulated by T-bet, were detected either in alveolar lymphocytes (IL-2R, CXCR3 and CXCR6) $[4,10,22]$ or in macrophages (CCL5 and CXCL10) [17, 19], we hypothesised that the same cell types may express Tbet. In our immunochemistry experiments, T-bet was localised to alveolar macrophages and lymphocytes and on lung biopsies to macrophages, multinucleated giant cells, epithelioid macrophages of the granuloma and lymphocytes. T-bet expression has been already detected in various cell types, such as natural killer, dendritic, and T- and B-cells [5]; hereby we report $\mathrm{T}$-bet expression also in lung macrophages. Interestingly, we noted enhanced production of T-bet in sarcoidosis patients with low BAL macrophages. Because we also detected upregulated CCL5 in these patients, we speculate that this fact may be a compensatory response to maintain the chemokine gradient of CCL5 in sarcoid lung. The dynamics of T-bet expression in alveolar macrophages during the disease course should be investigated in the future studies.

This study has limitations. First, we assessed only four patients with the fibrotic stage of sarcoidosis because of the limited number of patients available with this phenotype. Secondly, we used RT-PCR measurement of T-bet and chemokines/ receptors ex vivo to provide information about their possible relationship in sarcoidosis, but we did not prove the mechanism of interaction between T-bet and related genes by functional studies. Thirdly, this study only focused on the Tbet mRNA expression profiles in BAL cells and not in lung and 
lymph node tissues and peripheral blood cells. However, based on our immunohistochemistry experiments, it is evident that T-bet is also expressed in sarcoidosis-associated cells infiltrating sarcoid lung tissues and it should be investigated in future studies to obtain the complete picture of T-bet expression cells in sarcoidosis.

In conclusion, our data showed that expression of the Th1 transcription factor T-bet is upregulated in pulmonary sarcoidosis. A strong positive correlation was observed between Tbet and gene expression of IFN- $\gamma$ and CXCR3 in sarcoid BAL cells, suggesting previously reported direct control of these genes by T-bet also occurs in the lung compartment in sarcoidosis. Moreover, our correlation analysis revealed a relationship between T-bet and sarcoidosis-associated chemokines CCL5 and CXCL10, and receptors IL-2R/IL-15R and CXCR6 in sarcoid BAL cells. Further functional studies are needed to prove the mechanism of interaction between T-bet and related genes.

\section{SUPPORT STATEMENT}

Funding was obtained from the Czech Ministry of Health (IGA MZ CR NT/11117-6 and IGA MZ CR NR/9037) and, in part, from the Internal Grant Agency of Palacky University (IGA PU project SV LF_2010_008) and CZ.1.05/2.1.00/01.0030.

\section{STATEMENT OF INTEREST}

None declared.

\section{ACKNOWLEDGEMENTS}

We acknowledge the help of the staff of the Dept of Respiratory Medicine, Bronchoscopy Division, (Faculty Hospital, Olomouc, Czech Republic), A. Arakelyan (Institute of Molecular Biology, Armenian National Academy of Sciences, Yerevan, Republic of Armenia) for statistical advice, J. Srovnal (Laboratory of Experimental Medicine, Institute of Molecular and Translational Medicine, Faculty of Medicine and Dentistry, Palacky University, Olomouc, Czech Republic) for measurements of RNA integrity, and R. Langerova (Laboratory of Immunogenomics and Immunoproteomics, Faculty of Medicine and Denistry, Palacky University) and E. Sedlakova (Clinical and Molecular Pathology, Faculty of Medicine and Dentistry, Palacky University) for technical assistance in immunohistochemical experiments.

\section{REFERENCES}

1 Baughman RP, Lower EE, du Bois RM. Sarcoidosis. Lancet 2003; 361: 1111-1118.

2 Lynch JP 3rd, White ES. Pulmonary sarcoidosis. In: Drent M, Costabel U, eds. Sarcoidosis. Eur Respir Mon 2005; 32: pp. 105-129.

3 Semenzato G, Bortoli M, Brunetta E, et al. Immunology and pathophysiology. In: Drent M, Costabel U, eds. Sarcoidosis. Eur Respir Mon 2005; 32: pp. 49-63.

4 Agostini C, Cabrelle A, Calabrese F, et al. Role for CXCR6 and its ligand CXCL16 in the pathogenesis of T-cell alveolitis in sarcoidosis. Am J Respir Crit Care Med 2005; 172: 1290-1298.

5 Beima KM, Miazgowicz MM, Lewis MD, et al. T-bet binding to newly identified target gene promoters is cell-type independent, but results in variable context-dependent functional effects. J Biol Chem 2006; 281: 11992-12000.

6 Lewis MD, Miller SA, Miazgowicz MM, et al. T-bet's ability to regulate individual target genes requires the conserved T-box domain to recruit histone methyltransferase activity and a separate family member-specific transactivation domain. Mol Cell Biol 2007; 27: 8510-8521.
7 Matsuda JL, George TC, Hagman J, et al. Temporal dissection of T-bet functions. J Immunol 2007; 178: 3457-3465.

8 Szabo SJ, Kim ST, Costa GL, et al. A novel transcription factor, T-bet, directs Th1 lineage commitment. Cell 2000; 100: 655-669.

9 Zissel G, Prasse A, Müller-Quernheim J. Sarcoidosis-immunopathogenetic concepts. Semin Respir Crit Care Med 2007; 28: 3-14.

10 Katchar K, Eklund A, Grunewald J. Expression of Th1 markers by lung accumulated $\mathrm{T}$ cells in pulmonary sarcoidosis. J Intern Med 2003; 254: 564-571.

11 Ko FW, Lun SW, Wong CK, et al. Decreased T-bet expression and changes in chemokine levels in adults with asthma. Clin Exp Immunol 2007; 147: 526-532.

12 Ramhorst R, Patel R, Corigliano A, et al. Induction of maternal tolerance to fetal alloantigens by RANTES production. Am J Reprod Immunol 2006; 56: 302-311.

13 Phoon RK, Kitching AR, Odobasic D, et al. T-bet deficiency attenuates renal injury in experimental crescentic glomerulonephritis. J Am Soc Nephrol 2008; 19: 477-485.

14 Busuttil A, Weigt SS, Keane MP, et al. CXCR3 ligands are augmented during the pathogenesis of pulmonary sarcoidosis. Eur Respir J 2009; 34: 676-686.

15 Nishioka Y, Manabe K, Kishi J, et al. CXCL9 and 11 in patients with pulmonary sarcoidosis: a role of alveolar macrophages. Clin Exp Immunol 2007; 149: 317-326.

16 Arakelyan A, Kriegova E, Kubistova Z, et al. Protein levels of CC chemokine ligand (CCL)15, CCL16 and macrophage stimulating protein in patients with sarcoidosis. Clin Exp Immunol 2009; 155: 457-465.

17 Agostini C, Cassatella M, Zambello R, et al. Involvement of the IP10 chemokine in sarcoid granulomatous reactions. J Immunol 1998; 161: 6413-6420.

18 Petrek M, Kolek V, Szotkowská J, et al. CC and C chemokine expression in pulmonary sarcoidosis. Eur Respir J 2002; 20: 1206-1212.

19 Petrek M, Pantelidis P, Southcott AM, et al. The source and role of RANTES in interstitial lung disease. Eur Respir J 1997; 10: 1207-1216.

20 Spagnolo P, Renzoni EA, Wells AU, et al. C-C chemokine receptor 2 and sarcoidosis: association with Lofgren's syndrome. Am J Respir Crit Care Med 2003; 168: 1162-1166.

21 Petrek M, Gibejova A, Drabek J, et al. CC chemokine receptor 5 (CCR5) mRNA expression in pulmonary sarcoidosis. Immunol Lett 2002; 80: 189-193.

22 Agostini C, Trentin L, Facco M, et al. Role of IL-15, IL-2, and their receptors in the development of $\mathrm{T}$ cell alveolitis in pulmonary sarcoidosis. J Immunol 1996; 157: 910-918.

23 Kriegova E, Fillerova R, Arakelyan A, et al. T-bet is augmented during the pathogenesis of pulmonary sarcoidosis. Eur Respir $J$ 2009; 34: Suppl. 53, 501s.

24 Petrek M, Kolek V. Subpopulace T-lymfocytů v bronchoalveolární lavážní tekutině u plicní sarkoidózy a jiných intersticiálních plicních nemocí [T-lymphocyte subpopulations in bronchoalveolar lavage in pulmonary sarcoidosis and other interstitial pulmonary diseases]. Cas Lek Ces 1993; 132: 365-368.

25 Statement on sarcoidosis. Joint statement of the American Thoracic Society, the European Respiratory Society and World Association of Sarcoidosis and Other Granulomatous Disorders. Am J Respir Crit Care Med 1999; 160: 736-755.

26 Ziegenhagen MW, Rothe ME, Schlaak M, et al. Bronchoalveolar and serological parameters reflecting the severity of sarcoidosis. Eur Respir J 2003; 21: 407-413.

27 Kriegova E, Arakelyan A, Fillerova R, et al. PSMB2 and RPL32 are suitable denominators to normalize gene expression profiles in bronchoalveolar cells. BMC Mol Biol 2008; 9: 69.

28 Vandesompele J, De Preter K, Pattyn F, et al. Accurate normalization of real-time quantitative RT-PCR data by geometric 
averaging of multiple internal control genes. Genome Biol 2002; 3: RESEARCH0034.

29 User Bulletin \#2. ABI Prism 7700 Sequence Detection System. Relative Quantitation of Gene Expression. December, 1997. Date last updated: October 2001. Date last accessed: September 4, 2011. www3.appliedbiosystems.com/cms/groups/mcb_support/documents/generaldocuments/cms_040980.pdf.

30 Robinson BW, McLemore TL, Crystal RG. Gamma interferon is spontaneously released by alveolar macrophages and lung $\mathrm{T}$ lymphocytes in patients with pulmonary sarcoidosis. J Clin Invest 1985; 75: 1488-1495.

31 Shigehara K, Shijubo N, Ohmichi M, et al. Enhanced mRNA expression of Th1 cytokines and IL-12 in active pulmonary sarcoidosis. Sarcoidosis Vasc Diffuse Lung Dis 2000; 17: 151-157.

32 Matsuoka K, Inoue N, Sato T, et al. T-bet upregulation and subsequent interleukin 12 stimulation are essential for induction of
Th1 mediated immunopathology in Crohn's disease. Gut 2004; 53: 1303-1308.

33 Jöhrens K, Anagnostopoulos I, Stein H. T-bet expression patterns in coeliac disease, cryptic and overt enteropathy-type T-cell lymphoma. Histopathology 2005; 47: 368-374.

34 Frisullo G, Nociti V, Iorio R, et al. Increased expression of T-bet in circulating B cells from a patient with multiple sclerosis and celiac disease. Hum Immunol 2008; 69: 837-839.

35 Nizri E, Irony-Tur-Sinai $\mathrm{M}$, Lory $\mathrm{O}$, et al. Activation of the cholinergic anti-inflammatory system by nicotine attenuates neuroinflammation via suppression of Th1 and Th17 responses. J Immunol 2009; 183: 6681-6688.

36 Sopori M. Effects of cigarette smoke on the immune system. Nat Rev Immunol 2002; 2: 372-377.

37 Giri JG, Anderson DM, Kumaki S, et al. IL-15, a novel T cell growth factor that shares activities and receptor components with IL-2. J Leukoc Biol 1995; 57: 763-766. 